\title{
Estimating Throughput Available to a Node in Wireless Ad-hoc Network
}

\author{
Yong Shyang Liaw, Arek Dadej, Aruna Jayasuriya \\ Institute for Telecommunications Research, University of South Australia \\ Yong.Liaw@postgrads.unisa.edu.au, \{Arek.Dadej,Aruna.Jayasuriya\}@unisa.edu.au
}

\begin{abstract}
We propose a new technique to estimate the throughput available to a wireless node in a multi-hop network. Our technique exploits the nearly linear relationship between throughput at a node and the channel occupancy contributed by that node. It provides estimates of available throughput on the basis of local measurements and neighbourhood information available locally at a node. This technique allows timely and non-intrusive estimation without active probing. It is shown by simulations that this new technique provides robust estimates of throughput available to a node in an ad hoc network environment characterised by numerous active neighbours and dependencies between the levels of node activity along a multi-hop path.
\end{abstract}

\section{Introduction}

Robust and timely estimation of available link bandwidth and/or throughput is a necessary component of effective flow/congestion control and quality of service support mechanisms such as admission control, QoS routing and resource reservation in both wireline and wireless networks. In wireless network such as adhoc network, the bandwidth available to a node at any given time is affected by fading and shadowing effects in the wireless channel, changes in the network topology, and variations in the background traffic generated by the surrounding (neighbouring) nodes. These effects, not present in wireline networks, make the available channel bandwidth estimation in wireless networks a challenging task. Particularly in wireless ad-hoc networks, characterised by dynamic topology and limited bandwidth, we need estimation technique that is timely, responsive to changes in the available bandwidth, and non-intrusive. All these requirements are not sufficiently well addressed in the existing bandwidth estimation techniques $[1,2,3]$ designed for wired networks.
A common metric used in capacity estimation in wireless networks describes the transmit rate of a packet $\left(R_{\mathrm{pkt}}\right)$, given by $1 / \mathrm{t}_{\mathrm{tx}}$, where $t_{\mathrm{tx}}$ is the time from when the packet is ready for transmission at the head of the queue to an acknowledgement that the packet has been received. In [4], $R_{\mathrm{pkt}}$ is measured and normalised as the estimate of wireless link throughput, and in [5], the permissible throughput is estimated as $\left(1-U_{\text {queue }}\right) \times R_{\mathrm{pkt}}$, where $U_{\text {queue }}$ is the queue utilization. Estimates based on the transmit rate of a packet, $R_{\mathrm{pkt}}$, do not account for the increase in contention when the node is near its saturation point, and its subsequent effect on the available throughput. Furthermore, the $R_{\mathrm{pkt}}$ is based only on successful transmissions of packets; packets discarded after maximum number of retransmission attempts are ignored. Hence, the use of $R_{\mathrm{pkt}}$ leads to an over-optimistic estimate of the throughput available to a node. The ATP (Ad-hoc Transport Protocol) proposed in [6] relies on intermediate nodes along a path to provide rate feedback. The rate feedback is computed as $1 /\left(t_{\text {tx }}+t_{\text {queue }}\right)$, where $t_{\text {queue }}$ is the queue delay (time spent by a packet in the queue).

\section{Estimation of Available Throughput}

\subsection{Definitions}

We define the following concepts and parameters. The proportion of time a node spends in acquiring channel and transmission will be denoted by $U_{\mathrm{tx}}$; this includes the channel contention/backoff periods, handshaking and the transmission time in IEEE 802.11, as shown in Fig. 1. Since after a successful transmission the node will re-enter the channel acquisition state whenever there is a packet in the transmission queue, $U_{\mathrm{tx}}$ can be seen as the proportion of time the transmission queue is not empty (i.e. queue utilization).

We define the channel occupancy $(U)$ as the proportion of time when, from the perspective of a given node, either the channel is sensed busy or the node's transmission queue is not empty. Hence, if the 
node does not perceive the system as being in the state described by $U$, the node and the channel in its neighbourhood are idle. Please also note that $U=U_{\mathrm{tx}}+$ $U_{\mathrm{nb}}$, where $U_{\mathrm{nb}}$ is the channel occupancy introduced by the neighbours when the transmission queue at a node in question is empty.

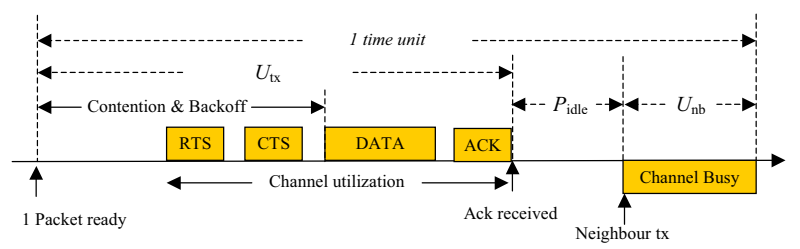

Figure 1. Channel activities for IEEE 802.11

\subsection{Throughput and Channel Occupancy}

To investigate how the total channel occupancy $U$ perceived by a node is related to the traffic contributed by that node, we ran simulations to measure the channel occupancy $(U)$ and throughput of a node $(T)$ with 7 active neighbours, at different levels of channel occupancy. The two $T-U$ graphs in Fig. 2 show that the throughput of a node is a nearly linear function of channel occupancy. The maximum throughput available to a node can therefore be estimated from (1) at the saturation point $U=1$. The degree of variation in the measured relationship, illustrated by the "thickness" of the $T-U$ graph, increases with the traffic load in the neighbourhood, mainly due to the effects of increasing channel contention. We will show that the slope $k$ may be estimated directly from the contribution of the node in question to the channel occupancy in the neighbourhood.

$$
T=k U-c=k\left(U-U_{z}\right), U_{z} \leq U \leq 1
$$

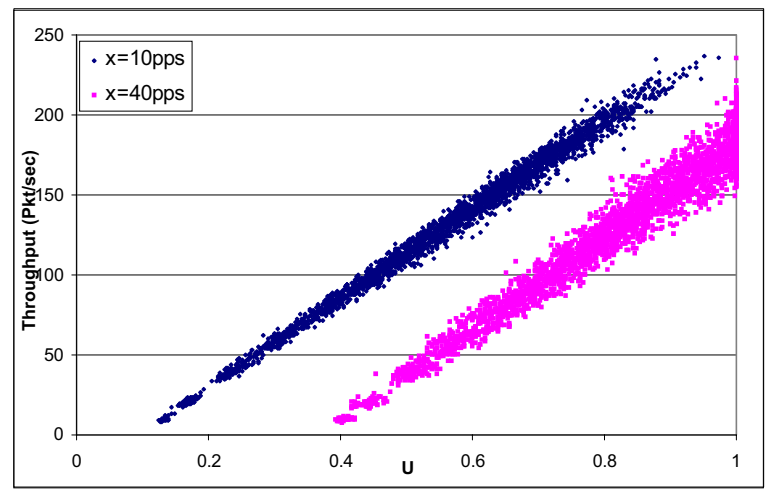

Figure 2. T-U graph for a node with 7 neighbours, each transmits at $x$ pkt/sec (pps)

\subsection{Throughput Available to a Node}

With the assumption of linear relationship between throughput and channel occupancy, we make a proportional estimate of the throughput available to a node. Let $x_{\mathrm{i}}$ be the throughput experienced by a Node-i. To correctly estimate the maximum throughput available to a node, we need to consider the dependencies between the levels of activity at nodes along the same path. In an ad-hoc network where traffic flows traverse multiple hops to their destinations, the traffic contributed by Node-i's neighbours while its transmit queue is empty (reflected by the $U_{\mathrm{nb}, \mathrm{i}}$ ) also includes traffic forwarded on its behalf (i.e. introduced by a proportion of Node-i's outgoing traffic), hence the traffic rates contributed by Node-i and its neighbours are inter-dependent. Let $\beta_{\mathrm{i}}$ be the proportion of $U_{\mathrm{nb}, \mathrm{i}}$ that is contributed by Node-i's outgoing traffic (i.e. the proportion of traffic forwarded by neighbours on behalf of Node-i). Then the total channel occupancy ( $\left.U_{\text {total,i }}\right)$ contributed directly and indirectly by Node- $i$ transmitting with throughput $x_{\mathrm{i}}$ is the sum $U_{\mathrm{tx}, \mathrm{i}}+$ $\beta_{i} \times U_{\mathrm{nb}, \mathrm{i}}$. With the remaining (unused) capacity of the channel described by the expression $\left(1-U_{\mathrm{i}}\right)$, the remaining throughput still available to a node is therefore $\left(1-U_{i}\right) \times x_{\mathrm{i}} / U_{\text {total,i, }}$, where the ratio $x_{\mathrm{i}} / U_{\text {total,i }}$ is the slope $k$ of the $T-U$ graph. The total throughput available to Node-i is obtained by adding its current throughput $x_{\mathrm{i}}$ to the remaining available throughput, and is given by (2).

$$
S T i=\left[\frac{1-U_{i}}{U_{t x, i}+\beta_{i} \times\left(U_{i}-U_{t x, i}\right)}+1\right] \times x_{i}
$$

To estimate $\beta_{\mathrm{i}}$, we assume as previously that the contribution to the channel occupancy by neighbouring nodes is proportional to the traffic rate they contribute. Hence $\beta_{\mathrm{i}}$ is the proportion of Node-i's neighbours' traffic that originates from Node-i's outgoing traffic,

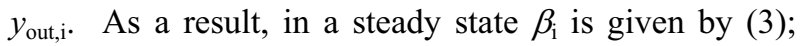
$e_{\text {out,i }}$ is the proportion of $y_{\text {out, }, \text { terminating at the next }}$ hop, $s_{\mathrm{i}}$ the traffic rate originating at Node-i and $J$ the set of Node-i's neighbours.

$$
\beta_{i}=\frac{\left(2-e_{\text {out }, i}\right) x_{i}-s_{i}}{\sum_{j \in J} x_{j}}
$$

\section{Simulations and Results}

We used Opnet simulations to investigate the accuracy of our estimates and compare them to those provided by other known techniques. DSR was used as the MANET routing protocol, and IEEE 802.11 as wireless $\mathrm{MAC}$ protocol, with fixed transmission range 
of 250 metres. The IEEE 802.11 was operated in DCF mode at $11 \mathrm{Mbps}$, with the RTS/CTS mechanism disabled. The data packet size was 1500 bytes. We collected relevant data (i.e. $U_{i}, U_{t x, i}, x_{i}$, etc), and then computed all other parameters of interest off-line for the network shown in Fig. 3. Here, $\beta_{0}$ is approximated by $x_{0} /\left(2 x_{0}+7 x\right)$, where $\mathrm{x}_{0}$ is the measured throughput of Node- 0 , and $x$ is the traffic rate of each neighbour.

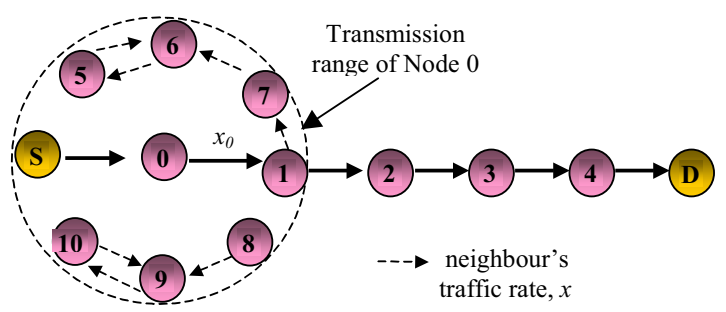

Figure 3. Network topology

Fig. 4-a confirms that the $T-U$ graphs are approximately linear, and the estimated saturation throughput is $115 \mathrm{pps}$ and $85 \mathrm{pps}$ for $\mathrm{x}=10 \mathrm{pps}$ and $30 \mathrm{pps}$ respectively. From Fig. 4-b and Fig. 4-c, the saturation throughput estimates (STE), as given by (2) for $\mathrm{x}=10 \mathrm{pps}$ and $30 \mathrm{pps}$, are close match to those obtained from the $T-U$ graph. For example, for $\mathrm{x}=30 \mathrm{pps}$ the STE remains fairly constant at $80 \mathrm{pps}(85 \mathrm{pps}$ from the $T-U$ graph) over the entire range of traffic rates at which the estimates were made. For $\mathrm{x}=10 \mathrm{pps}$, the STE is between $115 \mathrm{pps}$ and $135 \mathrm{pps}$ (115pps from the $T-U$ graph), with a maximum over-estimation error of $17.4 \%$.

As a comparison with other known techniques, Fig. 4-a and Fig. 4-c also show the total throughput (obtained by adding the permissible throughput [5] and current throughput) and the ATP feedback rate at $\mathrm{x}=10 \mathrm{pps}$ and 30pps. Clearly, both these techniques hugely overestimate the available throughput, particularly when the node in question is lightly loaded. Our technique gives a much better estimate over a wide range of traffic load/channel occupancy values.

\section{Conclusions}

We have proposed and described a new technique to estimate the throughput available to a node in a wireless multi-hop network, suitable for ad-hoc networks. This technique provides timely estimates and is non-intrusive, as it is based on local measurements and traffic rate information from neighbours. We have shown that this technique provides good estimates of throughput available to nodes in an ad-hoc network environment with numerous active neighbours and multi-hop paths.
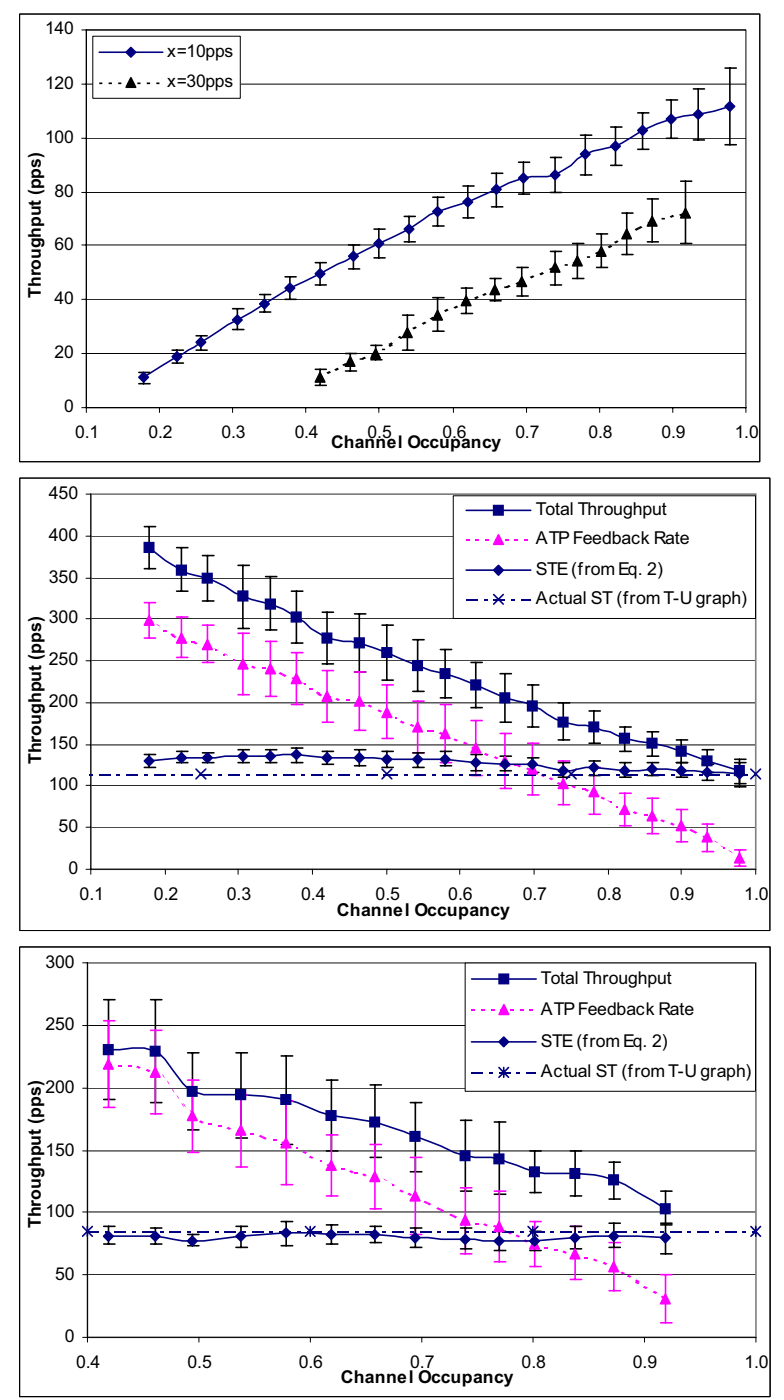

Figure 4. a) T-U Graph, Throughput estimates b) $x=10 p p s, c) x=30 p p s$

\section{References}

[1] Allen B. Downey, "Using pathchar to Estimate Internet Link Characteristics", ACM SIGCOMM, Aug 1999.

[2] Manish Jain, C. Dovrolis, "Pathload: A Measurement Tool for End-to-End Available Bandwidth", In Passive and Active Measurements, Fort Collin CO, March 2002.

[3] Kevin Lai, Mary Baker, "Measuring Bandwidth", Proc. Of IEEE INFOCOM, March 1999.

[4] Samarth H. Shah, Kai Chen, Klara Nahrstedt, "Dynamic Bandwidth Management for Single-hop Ad Hoc Wireless Networks", PerCom'03, 2003.

[5] Manthos Kazantzidis, Mario Gerla, "End-to-end versus Explicit Feedback Measurement in 802.11 Network", ISCC'02, 2002.

[6] Karthikeyan Sundaresan, et al, "ATP: A Reliable Transport Protocol for Ad-hoc Networks", Mobihoc'03, June 2003. 